\title{
A genetic variation of the transcription factor 7-like 2 gene is associated with risk of type 2 diabetes in the Japanese population
}

\author{
M. Horikoshi • K. Hara • C. Ito • R. Nagai • P. Froguel • \\ T. Kadowaki
}

Received: 9 August 2006 / Accepted: 4 December 2006 / Published online: 24 January 2007

(C) Springer-Verlag 2007

\begin{abstract}
Aims/hypothesis It has been suggested that transcription factor 7-like 2 protein (TCF7L2) plays an important role in glucose metabolism by regulating the production level of glucagon-like peptide-1, a hormone which modifies glucosedependent insulin secretion. Recently, variants of TCF7L2 gene were reported to confer an increased risk of type 2 diabetes in three different samples from European and European-origin populations. We studied whether the single nucleotide polymorphisms (SNPs) in TCF7L2 were associ-
\end{abstract}

M. Horikoshi and K. Hara contributed equally to the present study.

Electronic supplementary material The online version of this article (doi:10.1007/s00125-006-0588-6) contains supplementary material, which is available to authorised users.

\footnotetext{
M. Horikoshi $\cdot$ K. Hara $\cdot$ T. Kadowaki $(\bowtie)$

Department of Metabolic Diseases, Graduate School of Medicine, University of Tokyo,

Hongo 7-3-1, Bunkyo-ku,

Tokyo 113-8655, Japan

e-mail: kadowaki-3im@h.u-tokyo.ac.jp

M. Horikoshi $\cdot$ K. Hara $\cdot$ T. Kadowaki

CREST, Japan Science and Technology Corporation (JST),

Tokyo, Japan

C. Ito

Medical Court Life Care Clinic,

Hiroshima, Japan

R. Nagai

Department of Cardiovascular Medicine,

Graduate School of Medicine, University of Tokyo,

Tokyo, Japan

P. Froguel

Genomics and Molecular Physiology of Metabolic Diseases,

CNRS UMR,

8090 Lille Cedex, France
}

ated with type 2 diabetes in samples from a Japanese population.

Methods Five SNPs were genotyped in three different sample sets. Association with type 2 diabetes was investigated in each, as well as in combined sample sets.

Results The SNP rs7903146 was nominally associated with type 2 diabetes in the initial $(p=0.08)$ and two replication sample sets $(p=0.05$ and 0.06$)$. For the combined sample set, in which we successfully genotyped 1,174 type 2 diabetes patients and 823 control subjects, rs7903146 showed a significant association with type 2 diabetes (odds ratio $=1.69$ [95\% CI 1.21-2.36], $p=0.002$ ) with the same direction as the previous reports in samples from European and European-origin populations. SNPs rs7903146 and rs7901695 were in complete linkage disequilibrium. The rest of the five SNPs (rs7895340, rs11196205 and rs12255372) did not show any significant associations with type 2 diabetes.

Conclusions/interpretation The consistent association between rs7903146 in TCF7L2 and type 2 diabetes in different ethnic groups, including the Japanese population, suggests that TCF7L2 is a common susceptibility gene for type 2 diabetes.

Keywords Association - Susceptibility gene .

Type 2 diabetes

$\begin{array}{ll}\text { Abbreviations } \\ \text { GLP-1 } & \text { glucagon-like peptide-1 } \\ \text { HOMA } & \text { homeostasis model assessment } \\ \text { LD } & \text { linkage disequilibrium } \\ \text { OR } & \text { odds ratio } \\ \text { PAR } & \text { population attributable risk } \\ \text { SNP } & \text { single nucleotide polymorphism } \\ \text { TCF7L2 } & \text { transcription factor 7-like 2 }\end{array}$




\section{Introduction}

Transcription factor 7-like 2 protein (TCF7L2) regulates the production level of proglucagon, which is the precursor of the insulinotropic hormone glucagon-like peptide 1 (GLP-1) in enteroendocrine cells [1]. GLP-1 exerts critical effects on blood glucose homeostasis by increasing insulin secretion. TCF7L2 also has an essential role in the developmental and growth regulatory mechanisms of intestinal epithelial cells which secrete GLP-1, because TCF7L2-deficient mice lack an intestinal epithelial stem cell compartment [2]. TCF7L2 could influence susceptibility to type 2 diabetes by altering levels of GLP-1 and/or other hormones. Moreover, TCF7L2 is located in a chromosomal region that has been reported to be linked to type 2 diabetes in the Icelandic population [3]. Therefore, $T C F 7 L 2$ is a plausible candidate gene for type 2 diabetes. Recently, the Icelandic group reported that genetic variations located in introns of TCF7L2 were significantly associated with type 2 diabetes in samples from Icelandic, Danish and US populations [4].

In this study, we investigated whether the previously demonstrated genetic variations shown to be strongly associated with type 2 diabetes in the samples from European and European-origin populations are also associated with type 2 diabetes in samples from a Japanese population.

\section{Subjects and methods}

Subjects We performed association studies in three different sample sets (Table 1). In the initial and replication sample sets, diabetic patients were randomly recruited from among those attending the outpatient clinic of the Department of Metabolic Diseases, Graduate School of Medicine, University of Tokyo (Tokyo, Japan), and the non-diabetic subjects from among those undergoing annual health check-ups at the Hiroshima Atomic Bomb Casualty Council Health Management Center (Hiroshima, Japan). Another unrelated 356 diabetic patients and 192 control subjects were recruited from the same region and same facility in Hiroshima to avoid bias due to population stratification. The inclusion criteria for non-diabetic subjects were as follows: (1) $>60$ years of age; (2) $\mathrm{HbA}_{1 \mathrm{c}}$ values $<5.8 \%$; and (3) no family history of type 2 diabetes in first- and second-degree relatives. Diabetes was diagnosed in accordance with WHO criteria [5]. All participants gave informed consent, and the Ethics Committee of the University of Tokyo approved this study.

Single nucleotide polymorphism genotyping We genotyped all five TCF7L2 single nucleotide polymorphisms (SNPs) previously described by Grant et al. [4]. rs7901695 and rs7895340 were genotyped by direct sequencing, performed with a BigDye terminator (Applied Biosystems, Foster City, CA, USA) and resolved using an ABI 3700 automated DNA sequencer (Applied Biosystems). rs7903146, rs11196205 and rs12255372 were genotyped using Taqman SNP Genotyping Assays by means of an ABI 7900HT (Applied Biosystems) according to the manufacturer's protocol. Genotyping success rates were 99\% (rs7901695), 98\% (rs7903146), 94\% (rs7895340), 99\% (rs11196205) and 99\% (rs 12255372). Concordance rate, based on duplicate comparisons in 192 control subjects and 192 type 2 diabetes, was $100 \%$. All the SNPs were in accordance with HardyWeinberg equilibrium in type 2 diabetes subjects $(p>0.17)$, control subjects $(p>0.5)$ and the whole subject group $(p>0.13)$.

Biological measurements Insulin resistance and beta cell function were quantified using homeostasis model assessment (HOMA-IR and HOMA-beta, respectively); HOMA$\mathrm{IR}=($ fasting insulin $[\mathrm{pmol} / \mathrm{l}]) \times$ glucose $[\mathrm{mmol} / 1]) / 22.5 \times 6$ and HOMA-beta $=($ fasting insulin $[\mathrm{pmol} / 1]) \times 20) /($ glucose $[\mathrm{mmol} / \mathrm{l}]-3.5) \times 6$ as described elsewhere [6] (original equation modified to incorporate SI units, with $\mathrm{mU} / 1$ converted to $\mathrm{pmol} / \mathrm{l})$. Data were expressed as means $\pm \mathrm{SD}$.

Table 1 Clinical characteristics (means \pm SD) of the subjects

\begin{tabular}{|c|c|c|c|c|c|c|c|c|}
\hline & \multicolumn{2}{|l|}{ Initial } & \multicolumn{2}{|l|}{ Replication } & \multicolumn{2}{|l|}{ Hiroshima } & \multicolumn{2}{|c|}{ All subjects } \\
\hline & Diabetes & Control & Diabetes & Control & Diabetes & Control & Diabetes & Control \\
\hline$n$ & 192 & 272 & 657 & 360 & 356 & 192 & 1,205 & 824 \\
\hline Male & 123 & 129 & 421 & 141 & 217 & 93 & 761 & 363 \\
\hline Female & 69 & 143 & 236 & 219 & 139 & 99 & 444 & 461 \\
\hline Age at onset of diabetes (years) & $48.4 \pm 11.2$ & NA & $49.8 \pm 10.9$ & NA & $57.5 \pm 11.0$ & NA & $51.9 \pm 11.6$ & NA \\
\hline Age at examination (years) & $62.8 \pm 9.6$ & $68.6 \pm 6.7$ & $63.2 \pm 9.4$ & $70.5 \pm 6.8$ & $70.6 \pm 7.1$ & $68.6 \pm 6.7$ & $65.3 \pm 9.4$ & $69.4 \pm 6.8$ \\
\hline BMI $\left(\mathrm{kg} / \mathrm{m}^{2}\right)$ & $23.9 \pm 3.9$ & $24.0 \pm 3.8$ & $24.4 \pm 3.9$ & $23.6 \pm 3.5$ & $24.0 \pm 3.3$ & $23.8 \pm 4.0$ & $24.2 \pm 3.7$ & $23.8 \pm 3.7$ \\
\hline Fasting glucose $(\mathrm{mmol} / \mathrm{l})$ & $8.9 \pm 3.0$ & $5.2 \pm 0.5$ & $8.9 \pm 3.0$ & $5.0 \pm 0.8$ & $8.0 \pm 3.2$ & $5.2 \pm 0.9$ & $8.6 \pm 3.1$ & $5.1 \pm 0.7$ \\
\hline $\mathrm{HbA}_{1 \mathrm{c}}(\%)$ & $7.4 \pm 1.4$ & $5.2 \pm 0.3$ & $7.6 \pm 1.7$ & $5.2 \pm 0.2$ & $6.9 \pm 1.2$ & $5.2 \pm 0.2$ & $7.4 \pm 1.6$ & $5.2 \pm 0.2$ \\
\hline
\end{tabular}

NA Not applicable 
Statistical analysis The proportions of genotypes or alleles were compared between type 2 diabetic and non-diabetic subjects using a multiple logistic regression analysis adjusted for age, sex and BMI. Differences in HOMA according to genotypes were determined by analysis of covariates in nondiabetic subjects, after adjustment for age, sex and BMI. The statistical analyses were performed using JMP for Windows version 4.00 software (SAS Institute, Cary, NC, USA). To examine the pairwise linkage disequilibrium (LD) structure, $r^{2}$ between the SNPs were estimated via the method of maximum likelihood from two-locus genotype data using the expectation-maximisation algorithm under the assumption of Hardy-Weinberg equilibrium. The calculations were performed with SNPAlyze v3.2 Pro software (Dynacom, Yokohama, Japan). We considered $p<0.05$ to be significant. The odds ratio (OR) was assessed by counting the number of risk alleles for each individual, and we used the number of risk alleles to predict case/control status using logistic regression. Population attributable risk (PAR) was calculated as $\mathrm{PAR}=(p[\mathrm{OR}-1]) /(1+p[\mathrm{OR}-1])$, where $p$ is the prevalence of subjects with the risk allele.

\section{Results}

Genotype and allele frequencies of the five SNPs in TCF7L2 are shown in Table 2. Genotype frequency of rs7903146 was nominally associated with type 2 diabetes in each sample set $(p=0.08,0.05,0.06$; initial, replication and Hiroshima sample sets, respectively). However, when all the samples were combined, both genotype and minor allele frequencies were significantly associated with type 2 diabetes (OR 1.69 [95\% CI 1.21-2.36], $p=0.0075$ and $p=0.002$; genotype and minor allele frequencies, respectively). This significant association did not change when adjustment for BMI was omitted from the multiple logistic regression analysis (data not shown). The minor allele frequency of rs7903146 in the samples from a Japanese population (0.03-0.05) was substantially smaller than that of the previously reported three samples from European and European-origin populations, in which minor allele frequencies ranged from 0.27 to 0.39 . Association between rs 7903146 and type 2 diabetes in a dominant model was also examined, as its minor allele frequency was very low, but did not reach significance in two of our three sample sets. However, when all the samples were combined, we could confirm the significantly increased risk of type 2 diabetes in subjects with CT or TT (10.5 vs $6.4 \%$, type 2 diabetes vs controls, respectively; OR 1.75 [95\% CI 1.23-2.48], $p=0.0018$ ).

We found a significant interaction between SNP rs7903146 and BMI $(p=0.031)$ in the logistic regression analysis, suggesting that the effect of SNP rs7903146 on the risk of type 2 diabetes was different according to BMI. When we restricted the subjects to those with BMI lower than the median $\left(\mathrm{BMI}<23.8\right.$ and $<23.5 \mathrm{~kg} / \mathrm{m}^{2}$, for type 2 diabetes patients and control subjects, respectively), rs7903146 showed a higher OR (2.02 [95\% CI 1.28-3.21], $p=0.0027$; Electronic supplementary Table 1). The association was negative in subjects with BMI higher than the median (OR 1.32 [95\% CI 0.81-2.17], $p=0.27$ ).

rs7901695 showed a significant association with type 2 diabetes in the initial sample set (minor allele frequency: diabetes/control; 0.07/0.04, $p=0.04$ ), and because it was in complete LD $\left(r^{2}=1.0\right.$; Electronic supplementary Fig. 1) with rs7903146, we did not conduct further genotyping in the replication and Hiroshima sample sets. There were no significant differences in genotype or allele frequencies between type 2 diabetes patients and control subjects regarding rs7895340, rs11196205 and rs12255372 (Table 2).

We tested the SNP rs7903146 for quantitative trait association in non-diabetic subjects. rs7903146 did not show any association with age, sex, BMI and other clinical parameters related to type 2 diabetes such as $\mathrm{HbA}_{1 \mathrm{c}}$, fasting glucose, fasting insulin, HOMA-IR (CC vs CT/TT; $1.83 \pm 1.2$ vs $1.65 \pm 0.8, p=0.25$ ), and HOMA-beta (CC vs CT/TT; $100.0 \pm 65.7$ vs $97.6 \pm 49.8, p=0.79)$ in the non-diabetic subjects.

\section{Discussion}

In this study, we found a significant association in samples from a Japanese population between the variation in TCF $7 L 2$ and type 2 diabetes, an association similar to that previously reported in samples from European and European-origin populations $[4,7-13]$. It is noteworthy that the association between the SNP in TCF7L2 and type 2 diabetes has consistently been observed in different ethnic groups [14, 15], which supports the reliability of both previous studies as well as our present study. The mechanism of action of TCF7L2 in glucose metabolism and the pathogenesis of type 2 diabetes has yet to be elucidated, but it is possible that TCF7L2 has a role in regulating glucose-sensitive insulin secretion from beta cells. The prevalence of type 2 diabetes in the Japanese population is as high as in the USA [16], although the prevalence of obesity is much lower than that seen in Western countries [17]. One of the possible explanations is that fewer Japanese than European subjects are able to secrete enough insulin to compensate adequately for insulin resistance due to obesity [18]. Therefore, it is important to clarify the genetic components of susceptibility to insulin deficiency. Based on the present results, subjects having an at-risk allele account for $4 \%$ of the population, and the corresponding PAR is 3\%, a value much lower than that in samples from European and European-origin populations 


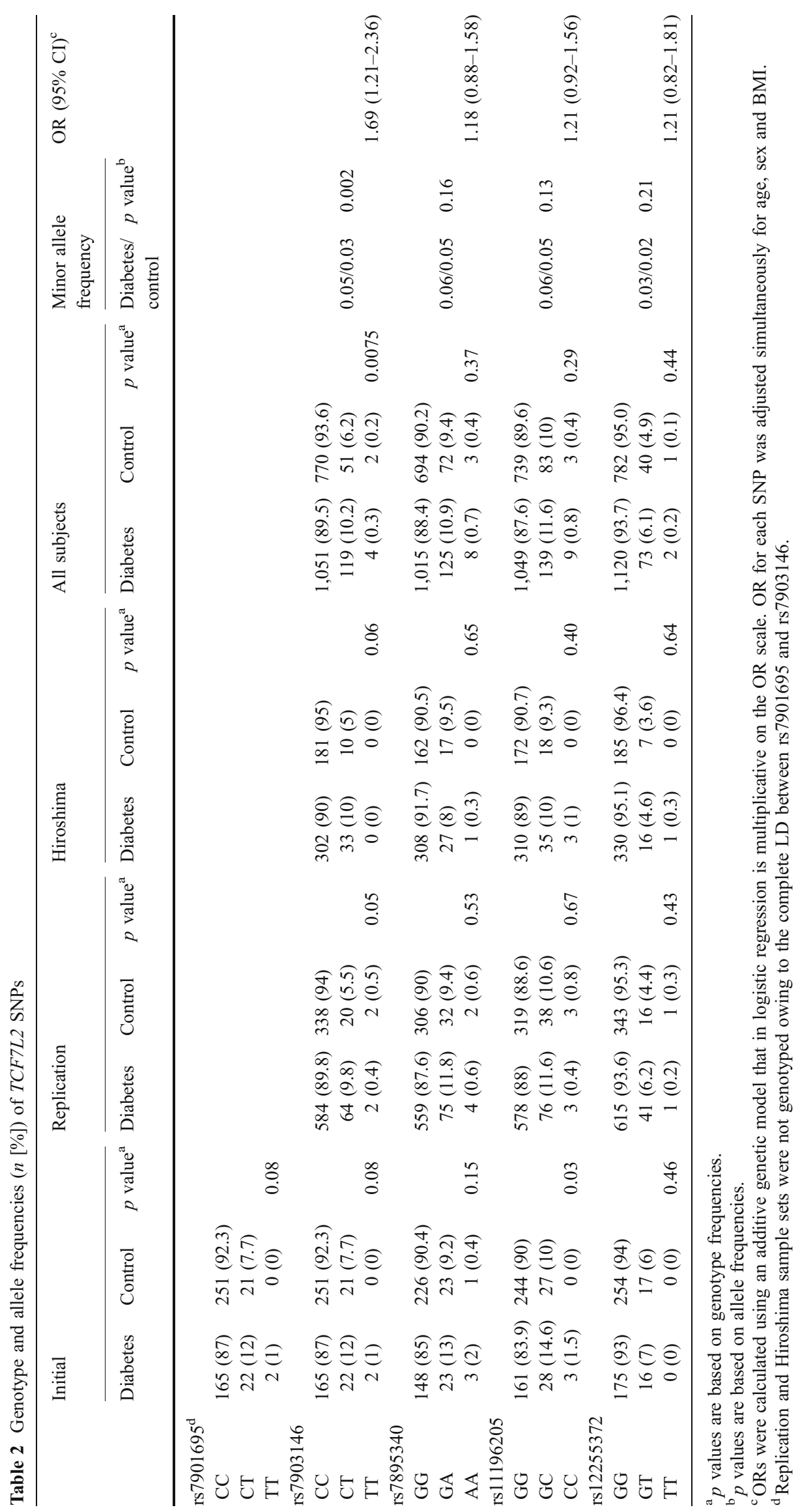


(21\%) [4]. It is possible that $T C F 7 L 2$ plays a substantial role in genetic susceptibility to insulin deficiency in the Japanese population. Florez et al. [19] reported that TCF7L2 polymorphisms were associated with increased risk of developing type 2 diabetes in samples from a population of European origins. In that study, associations between TCF7L2 polymorphisms and type 2 diabetes in other ethnic groups including Asians were also investigated. However, no significant associations were identified, possibly due to the small sample size. The present study provides important information suggesting $T C F 7 L 2$ is a type 2 diabetes susceptibility gene common to various ethnic groups including Japanese.

Acknowledgement This work was supported by a Grant-in-aid (to T. Kadowaki) from the Organization for Pharmaceutical Safety and Research and by a Grant-in-aid for the 21 st Century COE Program (to R. Nagai). We thank Y. Miyama for her technical assistance.

Duality of interest There is no duality of interest.

\section{References}

1. Yi F, Brubaker PL, Jin T (2005) TCF-4 mediates cell type-specific regulation of proglucagon gene expression by beta-catenin and glycogen synthase kinase-3beta. J Biol Chem 280:1457-1464

2. Korinek V, Barker N, Moerer P et al (1998) Depletion of epithelial stem-cell compartments in the small intestine of mice lacking Tcf4. Nat Genet 19:379-383

3. Reynisdottir I, Thorleifsson G, Benediktsson R et al (2003) Localization of a susceptibility gene for type 2 diabetes to chromosome 5q34-q35.2. Am J Hum Genet 73:323-325

4. Grant SF, Thorleifsson G, Reynisdottir I et al (2006) Variant of transcription factor 7-like 2 (TCF7L2) gene confers risk of type 2 diabetes. Nat Genet 38:320-322

5. Expert Committee on the Diagnosis and Classification of Diabetes Mellitus (1997) Report of the Expert Committee on the Diagnosis and Classification of Diabetes Mellitus. Diabetes Care 20:1183-1197

6. Matthews DR, Hosker JP, Rudenski AS et al (1985) Homeostasis model assessment: insulin resistance and beta-cell function from fasting plasma glucose and insulin concentrations in man. Diabetologia 28:412-419

7. Groves CJ, Zeggini E, Minton J (2006) Association analysis of 6,736 U.K. subjects provides replication and confirms TCF7L2 as a type 2 diabetes susceptibility gene with a substantial effect on individual risk. Diabetes 55:2640-2644

8. Zhang C, Qi L, Hunter DJ (2006) Variant of transcription factor 7-like 2 (TCF7L2) gene and the risk of type 2 diabetes in large cohorts of U.S. women and men. Diabetes 55:2645-2648

9. Scott LJ, Bonnycastle LL, Willer CJ et al (2006) Association of transcription factor 7-like 2 (TCF7L2) variants with type 2 diabetes in a Finnish sample. Diabetes 55:2649-2653

10. Damcott CM, Pollin TI, Reinhart LJ et al (2006) Polymorphisms in the transcription factor 7-like 2 (TCF7L2) gene are associated with type 2 diabetes in the Amish: replication and evidence for a role in both insulin secretion and insulin resistance. Diabetes 55:2654-2659

11. Saxena R, Gianniny L, Burtt NP et al (2006) Common single nucleotide polymorphisms in TCF7L2 are reproducibly associated with type 2 diabetes and reduce the insulin response to glucose in nondiabetic individuals. Diabetes 55:2890-2895

12. Cauchi S, Meyre D, Dina C et al (2006) Transcription factor TCF7L2 genetic study in the French population: expression in human beta-cells and adipose tissue and strong association with type 2 diabetes. Diabetes 55:2903-2908

13. van Vliet-Ostaptchouk JV, Shiri-Sverdlov R, Zhernakova A et al (2006) Association of variants of transcription factor 7-like 2 (TCF7L2) with susceptibility to type 2 diabetes in the Dutch Breda cohort. Diabetologia DOI 10.1007/s00125-006-0477-z

14. Humphries SE, Gable D, Cooper JA et al (2006) Common variants in the TCF7L2 gene and predisposition to type 2 diabetes in UK European Whites, Indian Asians and Afro-Caribbean men and women. J Mol Med 84(Suppl 1):1-10

15. Chandak GR, Janipalli CS, Bhaskar S et al (2006) Common variants in the TCF7L2 gene are strongly associated with type 2 diabetes mellitus in the Indian population. Diabetologia DOI 10.1007/s00125-006-0502-2

16. Wild S, Roglic G, Green A et al (2004) Global prevalence of diabetes. Diabetes Care 27:1047-1053

17. Yach D, Stuckler D, Brownell K (2006) Epidemiologic and economic consequences of the global epidemics of obesity and diabetes. Nat Med 12:62-66

18. Jensen CC, Cnop M, Hull RL et al (2002) Beta-cell function is a major contributor to oral glucose tolerance in high-risk relatives of four ethnic groups in the U.S. Diabetes 51:2170-2178

19. Florez JC, Jablonski KA, Bayley N et al (2006) TCF7L2 polymorphisms and progression to diabetes in the Diabetes Prevention Program. N Engl J Med 355:241-250 